\title{
A Rule Based Reasoning System For Initiating Passive ADAS Warnings Without Driving Distraction Through An Ontological Approach
}

\author{
Botao Fan \\ Bournemouth Univeristy \\ Bournemouth, UK \\ bfan@bournemouth.ac.uk
}

\author{
Jianbing Ma \\ Coventry University \\ Coventry, UK \\ ac1023@coventry.ac.uk
}

\author{
Nan Jiang \\ Bournemouth Univeristy \\ Bournemouth, UK \\ njiang@bournemouth.ac.uk
}

\author{
Huseyin Dogan \\ Bournemouth Univeristy \\ Bournemouth, UK \\ hdogan@bournemouth.ac.uk
}

\author{
Raian Ali \\ Bournemouth Univeristy \\ Bournemouth, UK \\ rali@bournemouth.ac.uk
}

\begin{abstract}
ADAS (Advanced Driver Assistance Systems) are in-vehicle systems designed to enhance driving safety and comfort. Unlike active ADAS which provide direct intervention to avoid accidents, passive ADAS increase driver's awareness of hazardous situations by giving warnings in advance. It has been noted that these systems can cause distraction when the relevant HMIs (Human-Machine Interfaces) are poorly designed. Current research is limited to address this problem in specific settings which may not be applicable in wider context. This papers aims to provide a universal rule-based solution to allow passive ADAS to initiate warnings without triggering driver distraction through an ontological approach.
\end{abstract}

Keywords-Advanced driver assistance system, driver distraction, Human-machine interface, Ontology

\section{INTRODUCTION}

Advanced driver assistance systems (ADAS) are a set of sensor based in-vehicle systems designed to help the driver in the driving process to enhance driving safety and comfort. [1]. ADAS can be briefly divided into two categories based on their roles in the driving process: active ADAS and passive ADAS [2]. Active ADAS, as the name implies, can provide direct intervention to help a driver avoid accidents in hazardous situations. Collision avoidance systems are good examples of active ADAS. These systems, equipped with autonomous braking, will apply partial of full braking force to stop the vehicle to avoid a collision if the driver ignores the warning or does not take action quickly enough against a detected hazard. In contrast, passive ADAS such as lane departure warning (LDW) and blind spot monitor (BSM) only provide a driver with needed information to increase their awareness to potential hazards. Obviously, unlike active ADAS, the effectiveness of passive ADAS relies on whether the driver can interpret the warning message correctly in a timely manner to allow them to take appropriate actions to avoid accidents.
With more sensor technologies become available inside a vehicle, there are concerns about driver information overload with automotive HMI which can lead to a concomitant increase in driver distraction [3]. This is because drivers need to process a large amount of information related to the primary driving task in the driving process (e.g., vehicle control) and they can easily get distracted when they are exposed to additional information sources requesting for the secondary tasks [3, 4]. Studies have noted that passive ADAS can become a major source of driving distraction which will compromise the primary driving task when their information provision is not designed carefully [4].Current research [5-11] has investigated some characteristics of passive ADAS warnings in order to improve their effectiveness but there still lacks a holistic understanding on how passive ADAS can trigger driver distraction in the context of driving tasks. This paper proposes a universal rule-based reasoning system to allow passive ADAS to initiate warnings without distracting drivers. This is done through an ontological approach which contextualizes the potential distractions associated with ADAS warnings and driving tasks.

This paper is organized as follows. The related work is presented in Section 2. This is followed by the ontology development in Section 3. Section 4 discusses how the rules can be created and used in real world scenarios. The conclusion is drawn in Section 5 where the suggestions for future research are also given.

\section{RELATED WORK}

Both active and passive ADAS rely on sensors to function so the evolvement of ADAS is always based on the development of sensor technologies. For example, Early ADAS (e.g., Anti-lock Braking System, ABS) were based on proprioceptive sensors which measure the internal status of the vehicle to enable the control of vehicle dynamics for enhanced safety [12]. The second generation of ADAS (e.g., Lane Departure Warning, LDW) was based on exteroceptive 
sensors which acquire information from outside the vehicle to enhance driving comfort. There are also more advanced ADAS based on sensor networks

There are different classifications of ADAS. Impactoriented classification provides a ranking of ADAS is based on a list of preselected road safety and traffic efficiency impact criteria [13]. These criteria include: (1) avoiding inappropriate speeds; (2) maintaining appropriate longitudinal and lateral distance; (3) supporting driver awareness; (4) speed adjustment; and (5) headway adjustment. Process oriented classification [14] features three categories based on the sub-processes driving ADAS are helping: perception, analysis-decision, and action. Because most ADAS rely on sensors to function, there are also classifications based on in-vehicle sensors (e.g., [15]). Figure 1 depicts a scenario of future communication technology in urban environments. Modern sensor technologies will generate more communication between vehicles and everything around them (e.g., other vehicles, the internet, and pedestrians). Such information could not only offer drivers a better understanding of the current situation but also help drivers make precise predictions of unseen events.

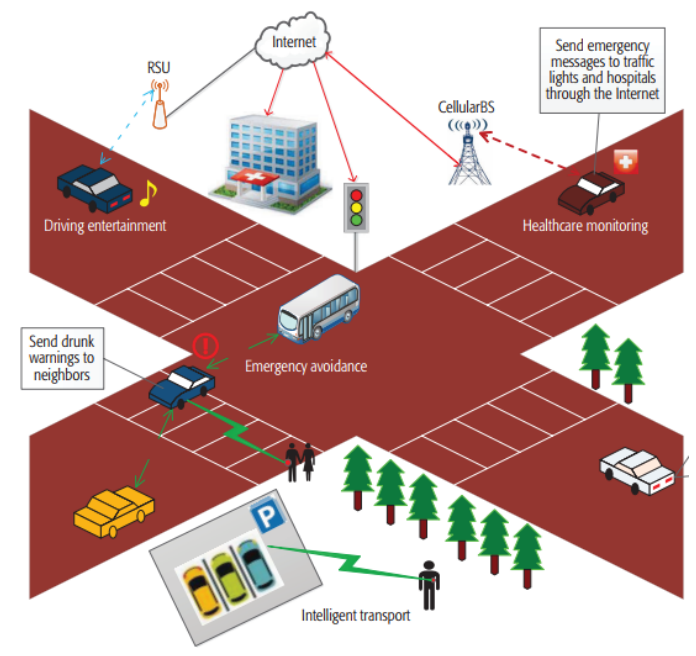

Fig. 1. A scenario of communication technology in urban environments [16]

Although ADAS aim to disburden drivers, such systems could become a major source of driving distraction because they provide more intensive information [17], especially in high visual demands and required complicated driverinitiated actions. Drivers must recognize the condition of the vehicle and the state of its surroundings through primarily visual (but also auditory and haptic) means and process this information in the brain, draw on memory to identify problem situations, decide on a plan of action, and execute it to avoid an accident. If there are too many simultaneous warnings across multiple ADAS, drivers may become confused about how to act, or they may just ignore the warnings or try to shut down the system. Perhaps, instead of performing actions themselves, drivers may just let the vehicle take evasion action.
Driver distraction is the primary cause of roadway injuries and fatalities [18]. A common understanding is that driver distraction is a subset of inattention where an explicit activity other than driving competes for the attention of the driver (e.g., operating a cell phone while driving) [19]. Driver distraction occurs when a triggering event induces an unintentional shift away from the primary task to affect driving safety [20-22]. Specifically, driving distraction involves four elements [23]:

- Delay by the driver in the recognition of information necessary to safely maintain lateral and longitudinal control of the vehicle (the driving task) (Impact)

- Due to some event, activity, object or person, within or outside the vehicle (Agent)

- That compels or tends to induce the driver's shifting attention away from fundamental driving tasks (Mechanism)

- By compromising the driver's auditory, biomechanical, cognitive or visual faculties, or combinations thereof (Type)

Passive ADAS deliver timely information regarding the surrounding driving environment and warnings about potential hazards through a Human-Machine Interface (HMI). The information is presented via three main modalities: visual, audio, and haptic. Traditionally, visual and auditory warnings have been used extensively in the design of ADAS information provision. Visual warnings are intuitional and can be used to convey various signals by symbolic information and color [24]. This modality is the fundamental strategy for information delivery. Visual warnings can be presented on the dashboard or center console of a vehicle but using these locations may potentially cause 'eye-off-road' lapses in driver attention. More recently, head-up displays have been used to present information and allow drivers to concentrate on the road $[25,26]$. However, such displays, as they are introduced to the driver's primary field of vision, may also interfere with their perception of the surrounding environment and increase reaction time [27]. Audio warnings are another type of warnings which usually produce faster responses than presenting visual warnings [28] and offer 'gaze-free' information [29]. A newer type of another modality is a haptic warning. Haptic warnings are suitable for alerting drivers about critical situations and helping them take corrective actions $[30,31]$. Also, the haptic modality can be used to present warnings to drivers without necessarily increasing their visual or auditory workload $[32,33]$.

Driving involves managing a high visual load with a variable cognitive load, leaving only limited resources for secondary task interactions [34]. Additional tasks (i.e., secondary tasks) are created by such in-vehicle systems, especially in passive ADAS, that could shift drivers' focus away from driving, and sometimes, they will simply not be able to resume the driving task quickly enough, which will substantially increase the risk of accidents [35]. To understand the nature of the interaction process' impact on driving, precisely defining the primary task is required. 
Primary driving task analysis falls under the larger purview of driver behavior models [36]. In the primary driving task, most of the information input is visual. The driver looks through the windscreen, checks the mirrors and blind spots, and glances at the instruments as required. Auditory and other sensory cues are available (such as the rumbling of a flat tire), but these play a minor role. The major outputs are hands and feet. The hands steer (lateral-directional), change gears, and operate various accessories [37], while the feet work the various pedals. However, the extant research has only concentrated on the action side of driving and neglected the driving context's impact. Drivers' behavior may be changed due to changes in the driving context. This conceptual shortcoming prompted the introduction of a taxonomy approach to the driving situation [38]. According to the authors' statement, the primary driving task has been divided into three components with corresponding types of roads: longitudinal, intersection, and other driving.

\section{ONTOLOGY-BASED APPROACH}

An ontology is a formal, explicit specification of a shared conceptualization [39]. It allows a shared and common understanding of some domain that can be communicated between people and application systems. An ontology involves with a formal description of concepts within a class (sometimes called a concept), descriptions of the features and attributes of the concept (or slots, sometimes called roles or properties), and restrictions on the slots (the facets, also known as role restrictions).

Developing an ontology emphasizes some fundamental rules: an iterative process where concepts should be close to the objects (physical or logical) and relationships in the domain of interest [40]. This ontology development lifecycle uses IDEF5 [41]. An IDEF5 development lifecycle is showed as following figure.

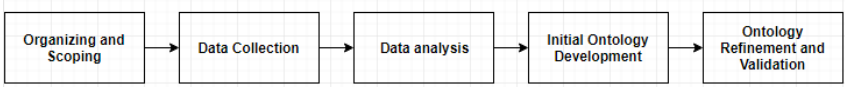

Fig. 2. IDEF5 development lifecycle

\section{A. Organizing and scoping}

To define the purpose and context of ontology, a number of competency (scoping) questions covering ADAS [42], driving tasks and driver distractions were determined as follows [43].

1) What objects can ADAS detect in the driving context?

2) What functions can be presented by ADAS?

3) What are the possible interactions between drivers and $A D A S$ ?

4) What are the primary driving tasks?

5) What types of distractions can occur during driving?

6) What are the impacts of different distractions on driving performance?

\section{B. Data Collection}

An extensive literature search on ADAS, driving tasks and driver distractions was performed where 17 source materials were identified for their high relevance. These include 8 journals, 6 conference papers, 2 websites and 1 ISO standard. As a result, 134 instances were extracted.

\section{Data Analysis}

After considering existing classifications/ontologies from the literature such as [44] and [38], an initial list of classes with references for ADAS was created as shown in Table 1.

TABLE I. CLASS AND REFERNCE RELATED TO ADAS

\begin{tabular}{|l|l|l|}
\hline Class & Sub-class & Reference \\
\hline $\begin{array}{l}\text { Environment } \\
\text { detection }\end{array}$ & $\begin{array}{l}\text { Road type, current line detection, } \\
\text { multi-lane detection, traffic light, } \\
\text { speed limit, weather conditions, and } \\
\text { light conditions. }\end{array}$ & {$[44][45]$} \\
\hline $\begin{array}{l}\text { Driver state } \\
\text { detection }\end{array}$ & Fatigue, driving ability & {$[46][47]$} \\
\hline $\begin{array}{l}\text { Ego-vehicle } \\
\text { detection }\end{array}$ & Velocity, orientation, and acceleration & {$[44]$} \\
\hline Free zone & Unmoving obstacles, navigable spaces & {$[44]$} \\
\hline $\begin{array}{l}\text { Moving } \\
\text { obstacles }\end{array}$ & $\begin{array}{l}\text { Other vehicles on the road, vulnerable } \\
\text { people }\end{array}$ & {$[44]$} \\
\hline Communication & $\begin{array}{l}\text { Other vehicles, road infrastructure, } \\
\text { pedestrians }\end{array}$ & {$[44][48]$} \\
\hline User input & $\begin{array}{l}\text { Buttons, touch screens, knobs, air- } \\
\text { gestures, audio comments }\end{array}$ & {$[49][50]$} \\
\hline Vehicle control & $\begin{array}{l}\text { Adjust speed, Automatically brake, } \\
\text { control steering, vision support }\end{array}$ & {$[51][52]$} \\
\hline Warnings & Modality, location, content & {$[53][54]$} \\
{$[25][55]$}
\end{tabular}

Similarly, classes for driving tasks and driver distraction were also created. Note that the classes for driving tasks were decided by considering the activities involved in the primary driving task such as following traffic and approaching junctions.

TABLE II. CLASS AND REFERENCE RELATED TO DRIVING TASK

\begin{tabular}{|l|l|l|}
\hline Class & Sub-class & Reference \\
\hline $\begin{array}{l}\text { Tasks performed during } \\
\text { longitudinal driving }\end{array}$ & $\begin{array}{l}\text { Free driving, following, } \\
\text { overtaking, being overtaken, } \\
\text { platoon driving, stop-and-go }\end{array}$ & {$[38]$} \\
\hline $\begin{array}{l}\text { Tasks performed in } \\
\text { intersections }\end{array}$ & $\begin{array}{l}\text { Intersection type, intersection } \\
\text { control, and driving direction }\end{array}$ & {$[38]$} \\
\hline Other driving tasks & $\begin{array}{l}\text { Reverse parallel parking and 3- } \\
\text { point turning }\end{array}$ & {$[56]$} \\
\hline Road characteristics & $\begin{array}{l}\text { Motorway, rural roadway, and } \\
\text { city roadway. }\end{array}$ & {$[38]$} \\
\hline
\end{tabular}

Table 3 shows the class and reference for driver distraction. As some of the driving distraction is acceptable [57], a class called duration is defined to indicate the length of a distraction for impact measurement. 
TABLE III. CLASS AND REFERENCE RELATED TO DRIVER DISTRACTION

\begin{tabular}{|l|l|l|}
\hline Class & Sub-class & Reference \\
\hline Type & Visual, audio, physical, cognitive & {$[58]$} \\
\hline Duration & Time & \\
\hline Impact & Eye off road, longitudinal impact, lateral impact & {$[59]$} \\
\hline
\end{tabular}

\section{Initial Ontology}

After renaming and removing some classes [38], the initial ontology was developed featuring three dimensions: ADAS, the driving task, and driver distraction. The main classes of ADAS include: data fusion, user interface (HMI) and vehicle control. The main classes are: driving straight, turning and slow maneuvers. The main classes are type, duration and impact as shown in Table 3.

\section{E. Ontology Refinement and Validation}

To refined and validate the initial ontology, a focus group study was considered where participants were asked to review the ontology in an open format. That is, they were allowed to modify, add and delete instances and classes based on their own driving experiences [60].

The focus group had eleven participants. They were $\mathrm{PhD}$ students and academics from the Department of Computing and Informatics, Bournemouth University. A pre-screening showed that six of them had been driving for more than ten years, two had five to ten years' driving experience, and three did not have a driving license but were taking driving lessons at the time the research was conducted. Table 4 shows the finalized ontology based on the focus group results.

TABLE IV. FINALIZED STRUCTURE OF EACH ONTOLOGY

\begin{tabular}{|c|c|c|}
\hline Ontology & Class & Sub-class \\
\hline \multirow[t]{12}{*}{ ADAS } & \multirow{5}{*}{$\begin{array}{l}\text { Sensor } \quad \text { Data } \\
\text { Fusion }\end{array}$} & Environment detection \\
\hline & & Driver state detection \\
\hline & & Ego-vehicle detection \\
\hline & & Free zone \\
\hline & & Moving obstacles \\
\hline & \multirow{5}{*}{$\begin{array}{l}\text { Interaction } \\
\text { Method }\end{array}$} & Button \\
\hline & & Touch screen \\
\hline & & Knob \\
\hline & & Air-gesture \\
\hline & & Audio commend \\
\hline & \multirow[t]{2}{*}{ Effect } & Warning \\
\hline & & Vehicle Control \\
\hline \multirow[t]{3}{*}{ Driving Task } & \multirow[t]{3}{*}{ Driving Straight } & Free driving, \\
\hline & & Following \\
\hline & & Overtaking \\
\hline
\end{tabular}

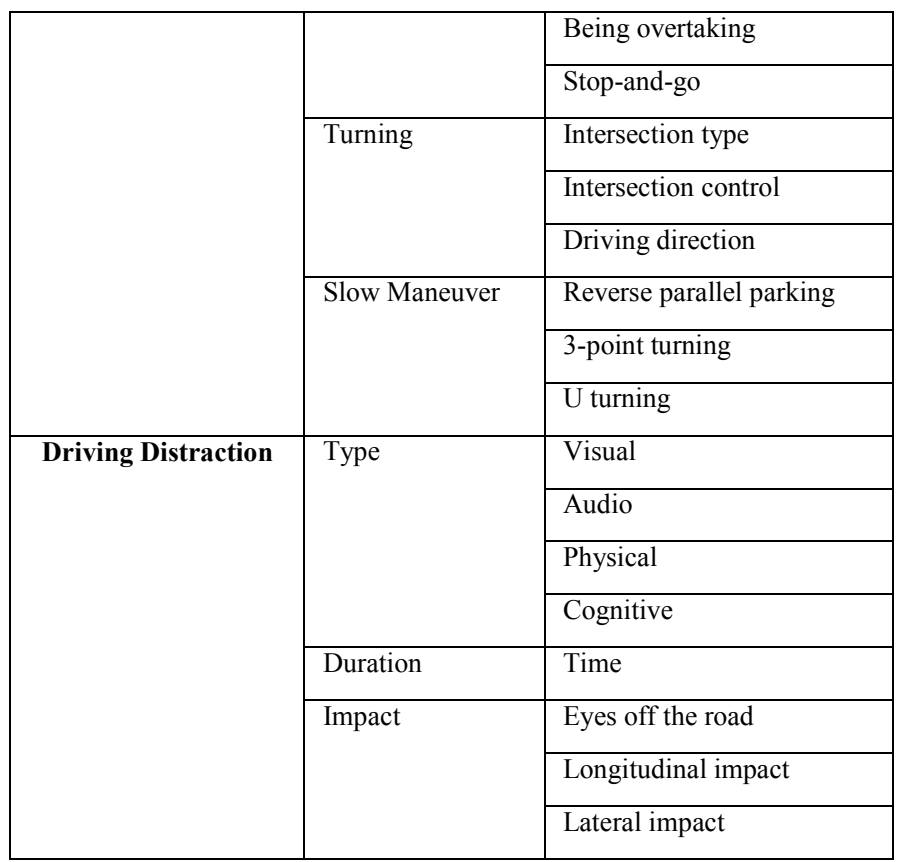

A schematic is created to indicate the process of how passive ADAS cause driver distraction. Sensor data determines ADAS warning. Drivers need time to notice and understand the meaning of warning. If such duration is greater than distraction guidelines, drivers may suffer from driver distraction (e.g. audio or visual) and have impact on their driving performance. The impact will finally affect current driving task.

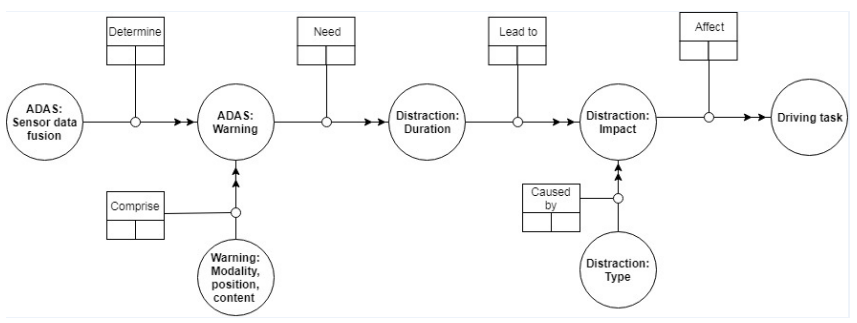

Fig. 3. Schematic diagram of how passive ADAS potentially cause driver distraction

\section{RULE-BASED WARNING INITIATION}

\section{A. Creating rules}

The main purpose of this research is to propose a universal rule-based reasoning system to enable passive ADAS warning initiation without distracting drivers. Therefore, the first step towards the system is to create fundamental rules based on the ontology developed. According to the relationship schematics of the ontology, each distraction situation has a relationship with the output data and accesses ADAS input data and driving tasks. Hence, the prerequisites for a reasoning system include matching entity classes to ontological classes, entity class relations to ontological binary relations between classes, and entity class attributes to ontological properties; these assign data types to ontological classes [61]. Rules are written in the Semantic Web Rule Language (SWRL). 
For demonstration purpose only, two rules are created in this section as examples. The first rule is used to determine the functionality of ADAS:

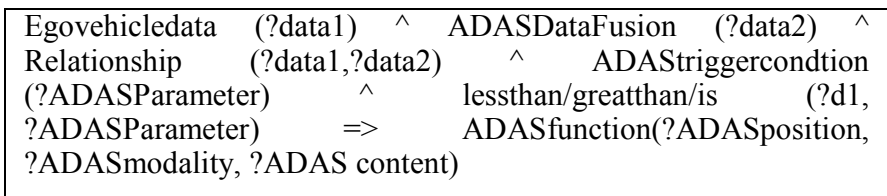

In this rule, ADAS detect ego-vehicle data and the surrounding driving environment (i.e. ?data2). These two pieces of data will have a relationship with each other, and the relationship will be compared with the ADAS' operational characteristics to determine the final output.

Following the driver's reaction to an ADAS function, the second rule is used to identify the potential driver distraction:

DistractionImpact (?Impact) $\wedge$ DistractionDuration (?Time) Distractionguideline (?Distractionparameter) $\wedge$ lessthan/greatthan/is (?Time, ?DistractionParameter) $=>$ Typedistraction (?Type)

\section{B. Reasoing Application}

A scenario is also created to demonstrate the use of the rule-based reasoning system (Figure 4). It should be noted that the scenario is based on the traffic conditions and regulations in the United Kingdom (UK). In this scenario, the subjective vehicle is following vehicle 1 in the built-up area where the speed limit is $30 \mathrm{MPH}$. The vehicle is equipped with forward collision warning (FCW) technology.

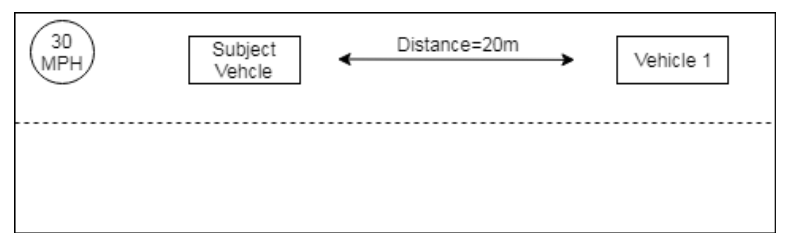

Fig. 4. A single warning scenario example

Rules can be transformed to the following:

Subvehicle $(? \mathrm{svX}) \wedge$ vehicle1 $(? \mathrm{v} 1 \mathrm{X}) \wedge$ Distancetosubvehicle $(? \mathrm{svX}, ? \mathrm{v} 1 \mathrm{X}, ? \mathrm{~d} 1) \wedge$ FCWtriggercondtion (?FCWParameter) $\wedge$ lessthan(?d1, ?FCWCParameter) $=>$ FWCwarning(?FCWposition, ?FCWmodality)

This rule determines the functionality of $\mathrm{FCW}$, which will be triggered when the distance between the two vehicles is less than a pre-defined threshold (e.g., 18 meters or 3 seconds). It is assumed that FWC provides a visual warning located on the dashboard. Then another rule can be created to identify potential distractions.

FocusonFCW (?eyeoffroad) $\wedge$ durationonFCW $($ ?time 1$) \wedge$
VisualdistractionParameter (?vdParameter) $\wedge$ greatThan $(?$ time 1,
?vdParameter) => Drivervisualdistraction $($ ?DVD)

As the driver will look at the dashboard when the FWC has been triggered, if the duration of the driver's visual focus on the dashboard is greater than a maximum time of 'eye off road' (e.g., 2 seconds [62]), a visual distraction will be identified and detected.

\section{CONCLUSION AND FUTURE WORK}

This work was the first attempt to provide a universal rule-based solution to enable passive ADAS to provide warnings without causing driver distraction. This was done by taking an ontological approach to understand how driving distraction can be triggered by passive ADAS in the context of driving tasks. After developing the ontology, two rules to determine the functionality of a passive ADAS and identify potential driver distraction based on driver's reaction to the ADAS warning were created. A singlewarning scenario was also given to demonstrate how the rule-based reasoning system could be used in a real world situation.

There are some limitations of the work. First, the developed ontology was only validated and refined with one focus group study involving only drivers. Clearly, a further study involving other stakeholders such as ADAS experts and automotive manufactures is needed to help improve the ontology. The refinement could be also looked into by considering drivers' experience and demographics as such factors can affect their attitudes. Second, the use of example rules was only demonstrated using a single warning scenario. In reality, there will be some scenarios involving multiwarnings (i.e. two or more warnings are triggered at the same time). Therefore, more scenarios focusing on multiwarnings should be created to verify the rules with the support of driving simulators. Third, only two rules were created as examples to show how the ontology could be used to help deduce rules. To proper assess the effectiveness of the reasoning system, more rules should be created in the future with appropriate driving scenarios.

\section{ACKNOWLEDGMENT}

This research is supported by Bournemouth University match-funded studentship: My navigation system really works for me - Designing ubiquitous in-car interfaces. The authors would also like to thank all academic and student volunteers for taking part in the focus group to help them refine and validate the ontology.

\section{REFERENCES}

[1] A. Tigadi, R. Gujanatti, A. Gonchi, and B. KLEMSSCET, "Advanced driver assistance systems," 2016.

[2] R. Troppmann, "Tech Tutorial: Driver Assistance Systems, an introduction to Adaptive Cruise Control: Part 1," https://www.embedded.com/print/4011081, 2006.

[3] J. L. Harbluk, Y. I. Noy, and M. Eizenman, "The impact of cognitive distraction on driver visual behaviour and vehicle control," 2002.

[4] A. Vahidi and A. Eskandarian, "Research advances in intelligent collision avoidance and adaptive cruise control," IEEE transactions on intelligent transportation systems, vol. 4, no. 3, pp. 143-153, 2003.

[5] Y.-C. Liu, "Effects of using head-up display in automobile context on attention demand and driving performance," Displays, vol. 24, no. 4, pp. 157-165, 2003.

[6] H. S. Park, M. W. Park, K. H. Won, K. H. Kim, and S. K. Jung, "In Vehicle AR - HUD System to Provide Driving - Safety Information," ETRI journal, vol. 35, no. 6, pp. 1038-1047, 2013. 
[7] K. Suzuki and H. Jansson, "An analysis of driver's steering behaviour during auditory or haptic warnings for the designing of lane departure warning system," JSAE review, vol. 24, no. 1, pp. 65-70, 2003.

[8] T. B. Sayer, J. R. Sayer, and J. M. Devonshire, "Assessment of a driver interface for lateral drift and curve speed warning systems: mixed results for auditory and haptic warnings," 2005.

[9] B. Forster, C. Cavina-Pratesi, S. M. Aglioti, and G. Berlucchi, "Redundant target effect and intersensory facilitation from visualtactile interactions in simple reaction time," Experimental brain research, vol. 143, no. 4, pp. 480-487, 2002.

[10] C. Ho, N. Reed, and C. Spence, "Multisensory in-car warning signals for collision avoidance," Human factors, vol. 49, no. 6, pp. 1107 $1114,2007$.

[11] F. Biondi, D. L. Strayer, R. Rossi, M. Gastaldi, and C. Mulatti, "Advanced driver assistance systems: Using multimodal redundant warnings to enhance road safety," Applied ergonomics, vol. 58, pp. 238-244, 2017.

[12] K. Bengler, K. Dietmayer, B. Farber, M. Maurer, C. Stiller, and H. Winner, "Three decades of driver assistance systems: Review and future perspectives," IEEE Intelligent Transportation Systems Magazine, vol. 6, no. 4, pp. 6-22, 2014.

[13] J. Golias, G. Yannis, and C. Antoniou, "Classification of driverassistance systems according to their impact on road safety and traffic efficiency," Transport reviews, vol. 22, no. 2, pp. 179-196, 2002.

[14] E. Rendon-Velez, "Classification and Overview of Advanced Driver Assistance Systems According to the Driving Process," in ASME 2010 International Design Engineering Technical Conferences and Computers and Information in Engineering Conference, 2010, pp. 687-695: American Society of Mechanical Engineers.

[15] M. Rezaei, M. Sarshar, and M. M. Sanaatiyan, "Toward next generation of driver assistance systems: A multimodal sensor-based platform," in Computer and Automation Engineering (ICCAE), 2010 The 2nd International Conference on, 2010, vol. 4, pp. 62-67: IEEE.

[16] W. Sun, J. Liu, and H. Zhang, "When smart wearables meet intelligent vehicles: challenges and future directions," IEEE wireless communications, vol. 24, no. 3, pp. 58-65, 2017.

[17] K. A. Brookhuis, D. De Waard, and W. H. Janssen, "Behavioural impacts of advanced driver assistance systems-an overview," European Journal of Transport and Infrastructure Research, vol. 1, no. 3, pp. 245-253, 2001.

[18] T. D. Johnson, "Distracted driving: Stay focused when on the road," 2012.

[19] J. T. Cohen and J. D. Graham, "A revised economic analysis of restrictions on the use of cell phones while driving," Risk Analysis, vol. 23, no. 1, pp. 5-17, 2003.

[20] F. M. Streff, "Driver distraction, aggression, and fatigue: a synthesis of the literature and guidelines for Michigan planning," 2000.

[21] K. Young, J. D. Lee, and M. A. Regan, Driver distraction: Theory, effects, and mitigation. CRC Press, 2008.

[22] J. D. Lee, K. Young, and M. A. Regan, Driver distraction: Theory, effects, and mitigation. CRC Press, 2008.

[23] M. Pettitt, G. E. Burnett, and A. Stevens, "Defining driver distraction," in 12th World Congress on Intelligent Transport SystemsITS AmericaITS JapanERTICO, 2005.

[24] C. C. Braun and N. C. Silver, "Interaction of signal word and colour on warning labels: differences in perceived hazard and behavioural compliance," Ergonomics, vol. 38, no. 11, pp. 2207-2220, 1995.

[25] H. Kim, A. Miranda Anon, T. Misu, N. Li, A. Tawari, and K. Fujimura, "Look at Me: Augmented Reality Pedestrian Warning System Using an In-Vehicle Volumetric Head Up Display," in Proceedings of the 21st International Conference on Intelligent User Interfaces, 2016, pp. 294-298: ACM.

[26] G. Jakus, C. Dicke, and J. Sodnik, "A user study of auditory, head-up and multi-modal displays in vehicles," Applied ergonomics, vol. 46, pp. 184-192, 2015.

[27] M. C. Schall and P. Gavin, "Attraction without distraction: Effects of augmented reality cues on driver hazard perception," 2010.
[28] S.-H. Chang, C.-Y. Lin, C.-P. Fung, J.-R. Hwang, and J.-L. Doong, "Driving performance assessment: effects of traffic accident location and alarm content," Accident Analysis \& Prevention, vol. 40, no. 5, pp. 1637-1643, 2008.

[29] R. Graham, "Use of auditory icons as emergency warnings: evaluation within a vehicle collision avoidance application," Ergonomics, vol. 42, no. 9, pp. 1233-1248, 1999.

[30] M. Enriquez and K. E. MacLean, "Impact of haptic warning signal reliability in a time-and-safety-critical task," in Haptic Interfaces for Virtual Environment and Teleoperator Systems, 2004. HAPTICS'04. Proceedings. 12th International Symposium on, 2004, pp. 407-414: IEEE.

[31] J. D. Lee, J. D. Hoffman, and E. Hayes, "Collision warning design to mitigate driver distraction," in Proceedings of the SIGCHI Conference on Human factors in Computing Systems, 2004, pp. 65 72: ACM.

[32] M. S. Prewett, L. R. Elliott, A. G. Walvoord, and M. D. Coovert, "A meta-analysis of vibrotactile and visual information displays for improving task performance," IEEE Transactions on Systems, Man, and Cybernetics, Part C (Applications and Reviews), vol. 42, no. 1, pp. 123-132, 2012.

[33] C. Spence and A. Gallace, "Recent developments in the study of tactile attention," Canadian Journal of Experimental Psychology/Revue canadienne de psychologie expérimentale, vol. 61, no. 3, p. 196, 2007.

[34] K. M. Bach, M. G. Jæger, M. B. Skov, and N. G. Thomassen, "Interacting with in-vehicle systems: understanding, measuring, and evaluating attention," in Proceedings of the 23rd British HCI Group Annual Conference on People and Computers: Celebrating People and Technology, 2009, pp. 453-462: British Computer Society.

[35] R. Häuslschmid, B. Pfleging, and A. Butz, "The Influence of Nondriving-Related Activities on the Driver's Resources and Performance," in Automotive User Interfaces: Springer, 2017, pp. 215-247.

[36] J. A. Michon, "A critical view of driver behavior models: what do we know, what should we do?," in Human behavior and traffic safety: Springer, 1985, pp. 485-524.

[37] W. W. Wierwille, "Visual and manual demands of in-car controls and displays," Automotive ergonomics, 1993.

[38] W. Fastenmeier and H. Gstalter, "Driving task analysis as a tool in traffic safety research and practice," Safety Science, vol. 45, no. 9, pp. 952-979, 2007.

[39] R. Studer, V. R. Benjamins, and D. Fensel, "Knowledge engineering: principles and methods," Data \& knowledge engineering, vol. 25, no. 1, pp. 161-197, 1998.

[40] N. F. Noy and D. L. McGuinness, "Ontology development 101: A guide to creating your first ontology," ed, 2001.

[41] D. Jones, T. Bench-Capon, and P. Visser, "Methodologies for ontology development," 1998.

[42] M. Grüninger and M. S. Fox, "Methodology for the Design and Evaluation of Ontologies," 1995.

[43] S. G. Klauer, T. A. Dingus, V. L. Neale, J. D. Sudweeks, and D. J. Ramsey, "The impact of driver inattention on near-crash/crash risk: An analysis using the 100-car naturalistic driving study data," 2006.

[44] E. Pollard, P. Morignot, and F. Nashashibi, "An ontology-based model to determine the automation level of an automated vehicle for co-driving," in Information Fusion (FUSION), 2013 16th International Conference on, 2013, pp. 596-603: IEEE.

[45] M.-Y. Fu and Y.-S. Huang, "A survey of traffic sign recognition," in Wavelet Analysis and Pattern Recognition (ICWAPR), 2010 International Conference on, 2010, pp. 119-124: IEEE.

[46] I. G. Daza, L. M. Bergasa, S. Bronte, J. J. Yebes, J. Almazán, and R. Arroyo, "Fusion of optimized indicators from Advanced Driver Assistance Systems (ADAS) for driver drowsiness detection," Sensors, vol. 14, no. 1, pp. 1106-1131, 2014.

[47] A. Koesdwiady, R. Soua, F. Karray, and M. S. Kamel, "Recent Trends in Driver Safety Monitoring Systems: State of the Art and Challenges," IEEE Transactions on Vehicular Technology, 2016. 
[48] S. Chen et al., "Vehicle-to-Everything (v2x) Services Supported by LTE-Based Systems and 5G," IEEE Communications Standards Magazine, vol. 1, no. 2, pp. 70-76, 2017.

[49] S. Damiani, E. Deregibus, and L. Andreone, "Driver-vehicle interfaces and interaction: where are they going?," European transport research review, vol. 1, no. 2, pp. 87-96, 2009.

[50] K. R. May, T. M. Gable, and B. N. Walker, "A multimodal air gesture interface for in vehicle menu navigation," in Adjunct Proceedings of the 6th International Conference on Automotive User Interfaces and Interactive Vehicular Applications, 2014, pp. 1-6: ACM.

[51] A. Paul, R. Chauhan, R. Srivastava, and M. Baruah, "Advanced Driver Assistance Systems," SAE Technical Paper0148-7191, 2016.

[52] A. Jefferson, "How Do Blind Spot Monitors Work?," 2015.

[53] E. Adell, A. Várhelyi, and M. Hjälmdahl, "Auditory and haptic systems for in-car speed management-A comparative real life study," Transportation research part F: traffic psychology and behaviour, vol. 11, no. 6, pp. 445-458, 2008.

[54] M. Lu, K. Wevers, and R. Van Der Heijden, "Technical feasibility of advanced driver assistance systems (ADAS) for road traffic safety," Transportation Planning and Technology, vol. 28, no. 3, pp. 167-187, 2005.

[55] ISO, "Road vehicles - Ergonomic aspects of in-vehicle presentation for transport information and control systems - Warning systems " 2005 .
[56] Mylicense.sa.go.au, "CBT\&A Task index," 2018

[57] S. Baker and K. Spina, "Drivers' attitudes, awareness and knowledge about driver distractions: Research from two central Sydney communities," 2007.

[58] K. Young, M. Regan, and M. Hammer, "Driver distraction: A review of the literature," Distracted driving, pp. 379-405, 2007.

[59] P. Papantoniou, E. Papadimitriou, and G. Yannis, "Review of driving performance parameters critical for distracted driving research," Transportation Research Procedia, vol. 25, pp. 1801-1810, 2017.

[60] J. Kitzinger, "Qualitative research. Introducing focus groups," BMJ: British medical journal, vol. 311, no. 7000, p. 299, 1995.

[61] M. Tonnis, G. Klinker, and J.-G. Fischer, "Ontology-based pervasive spatial knowledge for car driver assistance," in Pervasive Computing and Communications Workshops, 2007. PerCom Workshops' 07. Fifth Annual IEEE International Conference on, 2007, pp. 401-406: IEEE.

[62] B. G. Simons-Morton, F. Guo, S. G. Klauer, J. P. Ehsani, and A. K. Pradhan, "Keep your eyes on the road: Young driver crash risk increases according to duration of distraction," Journal of Adolescent Health, vol. 54, no. 5, pp. S61-S67, 2014. 